\title{
DEFORMATIONS OF STRUCTURES, EMBEDDING OF A RIEMANNIAN MANIFOLD IN A KÄHLERIAN ONE AND GEOMETRIC ANTIGRAVITATION
}

\author{
ALEXANDER A. ERMOLITSKI \\ Chair of Mathematics \\ Belorussian State Pedagogical University \\ Sovietskaya 18, Minsk 220050, Belarus \\ E-mail: erm@bspu.unibel.by
}

Tubular neighborhoods play an important role in modern differential topology. The main aim of the paper is to apply these constructions to geometry of structures on Riemannian manifolds. Deformations of tensor structures on a normal tubular neighborhood of a submanifold in a Riemannian manifold are considered in section 1 . In section 2 , this approach is used to obtain a Kählerian structure on the corresponding normal tubular neighborhood of the null section in the tangent bundle $T M$ of a smooth manifold $M$. In section 3, we consider a new deformation of a tensor structure on some neighborhood of a curve and introduce the so-called geometric antigravitation.

Some results of the paper were announced in [4], [5]. The work [3] is close to our discussion.

\section{Deformations of structures on a tubular neighborhood of a submanifold}

$\mathbf{1}^{\mathbf{0}}$. Let $\left(M^{\prime}, g^{\prime}\right)$ be a $k$-dimensional Riemannian manifold isometrically embedded in an $n$-dimensional Riemannian manifold $(M, g)$. The restriction of $g$ to $M^{\prime}$ coincides with $g^{\prime}$ and for any $p \in M^{\prime}$

$$
T_{p}(M)=T_{p}\left(M^{\prime}\right) \oplus T_{p}\left(M^{\prime}\right)^{\perp} .
$$

So, we obtain a vector bundle $M^{\prime} \rightarrow T\left(M^{\prime}\right)^{\perp}: p \mapsto T_{p}\left(M^{\prime}\right)^{\perp}$ over the submanifold $M^{\prime}$. There exists a neighborhood $\widetilde{U}_{0}$ of the null section $O_{M^{\prime}}$ in $T\left(M^{\prime}\right)^{\perp}$ such that the mapping

$$
\pi \times \exp : v \mapsto\left(\pi(v), \exp _{\pi(v)} v\right), v \in \widetilde{U}_{0}
$$

2000 Mathematics Subject Classification: 53C15, 53C26, 53C21.

Key words and phrases: Riemannian manifolds, almost Hermitian and tensor structures, tangent bundle.

The paper is in final form and no version of it will be published elsewhere. 
is a diffeomorphism of $\widetilde{U}_{0}$ onto an open subset $\widetilde{U} \subset M$. The subset $\widetilde{U}$ is called a tubular neighborhood of the submanifold $M^{\prime}$ in $M$. We assume that $\varepsilon$ is a positive number such that for any $p \in M^{\prime}$, the open geodesic balls $B\left(p ; \frac{\varepsilon}{2}\right) \subset B(p ; \varepsilon) \subset \widetilde{U}$. For example, such a number $\varepsilon$ exists if $M^{\prime}$ is compact. We denote $\widetilde{U}_{p}=\exp \left(\widetilde{U}_{0} \cap T_{p}\left(M^{\prime}\right)^{\perp}\right), D\left(p ; \frac{\varepsilon}{2}\right)=B\left(p ; \frac{\varepsilon}{2}\right) \cap$ $\widetilde{U}_{p}, D(p ; \varepsilon)=B(p ; \varepsilon) \cap \widetilde{U}_{p}$. It is clear that $\operatorname{dim} \widetilde{U}_{p}=\operatorname{dim} D\left(p ; \frac{\varepsilon}{2}\right)=\operatorname{dim} D(p ; \varepsilon)=n-k$. For any point $o \in M^{\prime}$, we can consider an orthonormal frame $\left(X_{1, o}, \ldots, X_{n, o}\right)$ such that $T_{o}\left(M^{\prime}\right)=\operatorname{Lin}\left[X_{1, o}, \ldots, X_{k, o}\right]$ and $T_{o}\left(M^{\prime}\right)^{\perp}=\operatorname{Lin}\left[X_{k+1, o}, \ldots, X_{n, o}\right]$. There exist coordinates $x_{1}, \ldots, x_{k}$ in some neighborhood $\widetilde{V}_{0} \subset M^{\prime}$ of the point $o$ such that $\frac{\partial}{\partial x_{i}} \mid o=X_{i, o}$, $i=1, \ldots, k$. We consider orthonormal vector fields $X_{k+1}, \ldots, X_{n}$ which are cross-sections of the vector bundle $p \mapsto T_{p}\left(M^{\prime}\right)^{\perp}$ over $\widetilde{V_{0}}$ and the neighborhood $\widetilde{W_{0}}=\bigcup_{p \in \widetilde{V}_{0}} \widetilde{U_{p}}$. The basis $\left\{X_{k+1, p}, \ldots, X_{n, p}\right\}$ defines the normal coordinates $x_{k+1}, \ldots, x_{n}$ on $\widetilde{U_{p}},[7]$. For any point $x \in \widetilde{W_{0}}$, there exists the unique point $p \in \widetilde{V_{0}}$ such that $x=\exp _{p}(t \xi),\|\xi\|=1$, $\xi \in T_{p}\left(M^{\prime}\right)^{\perp}$. A point $x \in \widetilde{W_{0}}$ has the coordinates $x_{1}, \ldots, x_{k}, x_{k+1}, \ldots, x_{n}$ where $x_{1}, \ldots, x_{k}$ are coordinates of the point $p$ in $\widetilde{V_{0}}$ and $x_{k+1}, \ldots, x_{n}$ are normal coordinates of $x$ in $\widetilde{U_{p}}$. We denote $X_{i}=\frac{\partial}{\partial x_{i}}, i=\overline{1, n}$, on $\widetilde{W_{0}}$. Thus, we can consider normal tubular neighborhoods $T b\left(M^{\prime} ; \frac{\varepsilon}{2}\right)=\bigcup_{p \in M^{\prime}} D\left(p ; \frac{\varepsilon}{2}\right)$ and $T b\left(M^{\prime} ; \varepsilon\right)=\bigcup_{p \in M^{\prime}} D(p ; \varepsilon)$ of the submanifold $M^{\prime}$.

$\mathbf{2}^{\mathbf{0}}$. Let $K$ be a smooth tensor field ot type $(r, s)$ on the manifold $M$ and for $x \in \widetilde{W_{0}}$, let

$$
K_{x}=\sum_{i_{1}, \ldots, i_{r}, j_{1}, \ldots, j_{s}} k_{j_{1}, \ldots, j_{s}}^{i_{1}, \ldots, i_{r}}(x) X_{i_{1}, x} \otimes \cdots \otimes X_{i_{r}, x} \otimes X_{x}^{j_{1}} \otimes \cdots \otimes X_{x}^{j_{s}},
$$

where $\left\{X_{x}^{1}, \ldots, X_{x}^{n}\right\}$ is the dual basis of $T_{x}^{*}(M), x=\exp _{p}(t \xi),\|\xi\|=1, \xi \in T_{p}\left(M^{\prime}\right)^{\perp}$. We define a tensor field $\bar{K}$ on $M$ in the following way.

a) $x \in D\left(p ; \frac{\varepsilon}{2}\right)$, then

$$
\overline{K_{x}}=\sum_{i_{1}, \ldots, i_{r}, j_{1}, \ldots, j_{s}} k_{j_{1}, \ldots, j_{s}}^{i_{1}, \ldots, i_{r}}(p) X_{i_{1}, x} \otimes \cdots \otimes X_{i_{r}, x} \otimes X_{x}^{j_{1}} \otimes \cdots \otimes X_{x}^{j_{s}}
$$

b) $x \in D(p ; \varepsilon) \backslash D\left(p ; \frac{\varepsilon}{2}\right)$, then

$$
\begin{array}{r}
\overline{K_{x}}=\sum_{i_{1}, \ldots, i_{r}, j_{1}, \ldots, j_{s}} k_{j_{1}, \ldots, j_{s}}^{i_{1}, \ldots, i_{r}}\left(\exp _{p}((2 t-\varepsilon) \xi)\right) X_{i_{1}, x} \\
\otimes \cdots \otimes X_{i_{r}, x} \otimes X^{j_{1} x} \otimes \cdots \otimes X_{x}^{j_{s}} ;
\end{array}
$$

c) $x \in M \backslash \bigcup_{M^{\prime}} D(p ; \varepsilon)$, then

$$
\overline{K_{x}}=K_{x} .
$$

It is easy to see that the tensor field $\bar{K}$ is independent of the choice of coordinates in $\widetilde{W}_{0}$ for every point $o \in M^{\prime}$.

Definition 1. The tensor field $\bar{K}$ is called a deformation of the tensor field $K$ on the normal tubular neighborhood of a submanifold $M^{\prime}$.

REMARK. The obtained tensor field $\bar{K}$ is continuous but it is not smooth on the boundaries of the normal tubular neighborhoods $T b\left(M^{\prime} ; \frac{\varepsilon}{2}\right)$ and $T b\left(M^{\prime} ; \varepsilon\right), \bar{K}$ is smooth in other points of the manifold $M$. 
$\mathbf{3}^{\mathbf{0}}$. We consider a deformation $\bar{g}$ of the Riemannian metric $g$ on the normal tubular neighborhood $T b\left(M^{\prime} ; \varepsilon\right)$ of a submanifold $M^{\prime}$. For $x \in \widetilde{W_{0}}, x=\exp _{p}(t \xi),\|\xi\|=1$, $\xi \in T_{p}\left(M^{\prime}\right)$, we define the Riemannian metric $\bar{g}$ in the following way.

a) $\overline{g_{p}}=g_{p}$ for any $p \in M^{\prime}$;

b) $\overline{g_{x}}\left(X_{i}, X_{j}\right)=\overline{g_{i j}}(x)=g_{i j}(p)$, where $X_{i}=\frac{\partial}{\partial x_{i}}, i=\overline{1, n}, X_{j}=\frac{\partial}{\partial x_{j}}, j=\overline{1, n}$, on $\widetilde{W}_{0}, x \in D\left(p ; \frac{\varepsilon}{2}\right)$;

c) $\overline{g_{x}}\left(X_{i}, X_{j}\right)=\overline{g_{i j}}(x)=\overline{g_{i j}}\left(\exp _{p}((2 t-\varepsilon) \xi)\right)$, for any $x \in D(p ; \varepsilon) / D\left(p ; \frac{\varepsilon}{2}\right)$;

d) $\overline{g_{x}}=g_{x}$ for each point $x \in M \backslash \bigcup_{p \in M^{\prime}} D(p ; \varepsilon)$.

The independence of $\bar{g}$ of a choice of local coordinates follows and the correctly defined Riemannian metric $\bar{g}$ on $M$ has been obtained.

It is known from [8] that every autoparallel submanifold of $M$ is a totally geodesic submanifold and a submanifold $M^{\prime}$ is autoparallel if and only if $\nabla_{X} Y \in T\left(M^{\prime}\right)$ for any $X, Y \in \chi\left(M^{\prime}\right)$, where $\nabla$ is the Riemannian connection of $\mathrm{g}$.

THEOREM 1. Let $M^{\prime}$ be a submanifold of a Riemannian manifold $(M, g)$ and $\bar{g}$ be the deformation of $g$ on the normal tubular neighborhood $T b\left(M^{\prime} ; \varepsilon\right)$ of $M^{\prime}$ constructed above. Then $M^{\prime}$ is a totally geodesic submanifold of $\left(T b\left(M^{\prime} ; \frac{\varepsilon}{2}\right), \bar{g}\right)$.

Proof. For any point $x \in D\left(p ; \frac{\varepsilon}{2}\right) \subset \widetilde{W_{0}}$ the functions $\bar{g}_{i j}(x)=g_{i j}(p)$ and $\frac{\partial \bar{g}_{i j}}{\partial x_{l}}=0, l=$ $\overline{k+1, n}$ on $D\left(p ; \frac{\varepsilon}{2}\right)$ because the vector fields $X_{l}=\frac{\partial}{\partial x_{l}}$ are tangent to $D\left(p ; \frac{\varepsilon}{2}\right)$. By the formula of the Riemannian connection $\bar{\nabla}$ of the Riemannian metric $\bar{g}$, [7], we obtain for $i, j=\overline{1, k}, l=\overline{k+1, n}$

$$
\begin{aligned}
& 2 \bar{g}_{p}\left(\bar{\nabla}_{X_{j}}, X_{j}, X_{l}\right)=X_{i_{p}} \bar{g}\left(X_{j}, X_{l}\right)+X_{j_{p}} \bar{g}\left(X_{i}, X_{l}\right) \\
& -X_{l_{p}} \bar{g}\left(X_{i}, X_{j}\right)+\bar{g}_{p}\left(\left[X_{i}, X_{j}\right], X_{l}\right)+\bar{g}_{p}\left(\left[X_{l}, X_{i}\right], X_{j}\right) \\
& +\bar{g}_{p}\left(X_{i},\left[X_{l}, X_{j}\right]\right)=-\frac{\partial \bar{g}_{i j}}{\partial x_{l}}=0 .
\end{aligned}
$$

Here we use the fact that $\left[X_{i}, X_{j}\right]=\left[X_{l}, X_{i}\right]=\left[X_{l}, X_{j}\right]=0$ and that $\bar{g}\left(X_{j}, X_{l}\right)=$ $\bar{g}\left(X_{i}, X_{l}\right)=0$ because $X_{l} \in T\left(M^{\prime}\right)^{\perp}$. Thus, $\bar{\nabla}_{X_{i}} X_{j} \in T\left(M^{\prime}\right)$ and from the remarks above the theorem follows.

COROllary 1. Let $\bar{R}$ be the Riemannian curvature tensor field of $\bar{g}$. Then $\bar{R}$ vanishes on every $D\left(p ; \frac{\varepsilon}{2}\right)$ for $p \in M^{\prime}$.

Proof. From the formula (1.1) it is clear that $\bar{\nabla}_{X_{l}} X_{m}=0$ for $l, m=\overline{k+1, n}$. The rest is obvious.

$4^{\mathbf{0}}$. Let $(F, \xi, \eta, g)$ be an almost contact metric structure (acms) on $M$ and $M^{\prime} \cong S^{1}$ be a closed integral curve of the vector field $\xi$ passing through a point $o \in M^{\prime}$. We consider a tubular neighborhood $\widetilde{U}$ of the submanifold $M^{\prime}$ and for any point $o \in M^{\prime}$ the coordinate neighborhood $\widetilde{W_{0}}=\bigcup_{p \in \widetilde{V}_{0}} \widetilde{U_{p}}$ with the coordinates $x_{1}, \ldots, x_{2 n}, x_{2 n+1}$ where $x_{2 n+1}$ is the coordinate of the point $p \in \widetilde{V_{0}} \subset M^{\prime}$ and $x_{1}, \ldots, x_{2 n}$ are normal coordinates of $x \in \widetilde{U_{0}}$, $x=\exp _{p}(t \nu),\|\nu\|=1, \nu \in T_{p}\left(M^{\prime}\right)^{\perp}$. If $X_{i}=\frac{\partial}{\partial x_{i}}, i=\overline{1,2 n+1}$ on $\widetilde{W_{0}}$, then $\frac{\partial}{\partial x_{2 n+1}}=\xi$ and $\frac{\partial}{\partial x_{\left.i\right|_{p}}}, i=\overline{1,2 n}$, are orthonormal vectors for any $p \in \widetilde{V_{0}},\left\{X_{1_{p}}, \ldots, X_{2 n p}\right\}$ define the 
normal coordinates $x_{1}, \ldots, x_{2 n}$ on $\widetilde{U}_{p}$, see $\mathbf{1}^{\mathbf{0}}$. We can choose cross-sections $X_{1}, \ldots, X_{2 n}$ of the vector bundle $p \mapsto T_{p}\left(M^{\prime}\right)^{\perp}$ over $\tilde{V}_{0}$ in such a way that $F X_{i}=X_{n+i}, i=\overline{1, n}$; $F X_{j}=-X_{j-n}, j=\overline{n+1,2 n}$. A deformation $\bar{g}$ of the Riemannian metric $g$ is defined on the normal tubular neighborhood $T b\left(M^{\prime} ; \varepsilon\right)$ of $M^{\prime}$, see $\mathbf{3}^{\mathbf{0}}$. Further, a deformation $\bar{F}$ of the $(1,1)$ tensor field $F$ on $M$ is defined in the following way.

a) $x \in D\left(p ; \frac{\varepsilon}{2}\right) \subset \tilde{U}_{p} \subset \tilde{W}_{0}$,

$$
\bar{F}_{x} X_{i}=F_{p} X_{i}, i=\overline{1,2 n} ; \bar{F}_{x} \xi=0 ;
$$

b) $x \in D(p ; \varepsilon) \backslash D\left(p ; \frac{\varepsilon}{2}\right)$,

$$
\begin{gathered}
x=\exp _{p}(t \nu),\|\nu\|=1, \nu \in T_{p}\left(M^{\prime}\right)^{\perp} ; \\
\bar{F}_{x} X_{i}=F_{\exp _{p}((2 t-\varepsilon) \nu)} X_{i}, i=\overline{1,2 n}, \bar{F}_{x} \xi=0 ;
\end{gathered}
$$

c) $x \in M \backslash \bigcup_{p \in M^{\prime}} D(p ; \varepsilon)$,

$$
\bar{F}_{x}=F_{x} .
$$

It is clear that $\bar{g}(\bar{F} X, \bar{F} Y)=\bar{g}(X, Y)$ for $X, Y \in \operatorname{Lin}[\xi]^{\perp}$ in the cases a), c). In the case b) we obtain

$$
\begin{aligned}
\bar{g}_{x}\left(\bar{F}_{x} X_{i}, \bar{F}_{x} X_{j}\right) & =g_{\exp _{p}((2 t-\varepsilon) \nu)}\left(F_{\exp _{p}((2 t-\varepsilon) \nu)} X_{i}, F_{\exp _{p}((2 t-\varepsilon) \nu)} X_{j}\right) \\
& =g_{\exp _{p}((2 t-\varepsilon) \nu)}\left(X_{i}, X_{j}\right)=\bar{g}_{x}\left(X_{i}, X_{j}\right) .
\end{aligned}
$$

If $\bar{\eta}(X)=\bar{g}(X, \xi)$ for $X \in \chi(M)$ then we have got the correctly defined acms $(\bar{F}, \xi, \bar{\eta}, \bar{g})$ on $M$.

Proposition 2. The curve $M^{\prime}$ is a geodesic with respect to $\bar{\nabla}$ and $D\left(p ; \frac{\varepsilon}{2}\right)$ is a flat totally geodesic submanifold of $\left(T b\left(M^{\prime} ; \frac{\varepsilon}{2}\right), \bar{g}\right)$ for any $p \in M^{\prime}$. The structure $(\bar{F}, \bar{g})$ is a Kählerian one on every $D\left(p ; \frac{\varepsilon}{2}\right), p \in M^{\prime}$.

Proof. The equality $\bar{\nabla}_{\xi_{p}} \xi=0$ directly follows from (1.1). From Corollary 1 we see that $\bar{\nabla}_{X_{i}} X_{j}=0$ on $D\left(p ; \frac{\varepsilon}{2}\right), i, j=\overline{1,2 n}$, and the first conclusion of the Proposition 2 is fulfilled. Further, we have

$$
\bar{\nabla}_{X_{i}} \bar{F} X_{j}-\bar{F} \bar{\nabla}_{X_{i}} X_{j}= \pm \nabla_{X_{i}} X_{l}=0
$$

where $l=n+j, j=\overline{1, n}$; or $l=j-n, j=\overline{n+1,2 n}$. So we obtain $\bar{\nabla} \bar{F}=0$ on $D\left(p ; \frac{\varepsilon}{2}\right)$ and $(\bar{F}, \bar{g})$ is a Kählerian structure on every $D\left(p ; \frac{\varepsilon}{2}\right), p \in M^{\prime}$.

\section{Embedding of a Riemannian manifold into a Kählerian one}

$\mathbf{1}^{\mathbf{0}}$. Let $(M, g)$ be an $n$-dimensional Riemannian manifold and $T M$ be its tangent bundle. For the Riemannian connection $\nabla$ we consider the connection mapping $K$ of $\nabla$ [1], [6], defined by the formula

$$
\nabla_{X} Z=K Z * X
$$

where $Z$ is considered as a mapping from $M$ into $T M$ and the right hand side means a vector field on $M$ assigning to $p \in M^{\prime}$ the vector $K Z_{*} X_{p} \in M_{p}=T_{p}(M)$.

If $U \in T M$, we denote by $H_{U}$ the kernel of $K_{\mid T M_{U}}$ and this $n$-dimensional subspace of $T M_{U}$ is called the horizontal subspace of $T M_{U}=T_{U}(T M)$. 
Let $\pi$ denote the natural projection of $T M$ onto $M$, then $\pi_{*}$ is a $C^{\infty}$-map of $T T M$ onto $T M$. If $U \in T M$, we denote by $V_{U}$ the kernel of $\pi_{* \mid T M_{U}}$ and this $n$-dimensional subspace of $T M_{U}$ is called the vertical subspace of $T M_{U}\left(\operatorname{dim} T M_{U}=2 \operatorname{dim} M=2 n\right)$. The following maps are isomorphisms of the corresponding vector spaces $(p=\pi(U))$

$$
\pi_{* \mid T M_{U}}: H_{U} \rightarrow M_{p}, K_{\mid T M_{U}}: V_{U} \rightarrow M_{p}
$$

and we have

$$
T M_{U}=H_{U} \oplus V_{U}
$$

If $X \in \chi(M)$, then there exists exactly one vector field on $T M$ called the "horizontal lift" (resp. "vertical lift") of $X$ and denoted by $\bar{X}^{h}\left(\bar{X}^{\nu}\right)$, such that for all $U \in T M$

$$
\begin{aligned}
& \pi_{*} \bar{X}_{U}^{h}=X_{\pi(U)}, K \bar{X}_{U}^{h}=0_{\pi(U)}, \\
& \pi_{*} \bar{X}_{U}^{v}=0_{\pi(U)}, K \bar{X}_{U}^{v}=X_{\pi(U)} .
\end{aligned}
$$

Let $R$ be the curvature tensor field of $\nabla$, then following [1] we have

$$
\begin{gathered}
{\left[\bar{X}^{\nu}, \bar{Y}^{\nu}\right]=0,} \\
{\left[\bar{X}^{h}, \bar{Y}^{\nu}\right]=\left(\overline{\nabla_{X} Y}\right)^{\nu},} \\
\pi_{*}\left(\left[\bar{X}^{h}, \bar{Y}^{h}\right]_{U}\right)=[X, Y], \\
K\left(\left[\bar{X}^{h}, \bar{Y}^{h}\right]_{U}\right)=R(X, Y) U .
\end{gathered}
$$

For vector fields $\bar{X}=\bar{X}^{h} \oplus \bar{X}^{\nu}$ and $\bar{Y}=\bar{Y}^{h} \oplus \bar{Y}^{\nu}$ on $T M$, the natural Riemannian metric $\hat{g}=<,>$ is defined on $T M$ by the formula

$$
<\bar{X}, \bar{Y}>=g\left(\pi_{*} \bar{X}, \pi_{*} \bar{Y}\right)+g(K \bar{X}, K \bar{Y}) .
$$

It is clear that the subspaces $H_{U}$ and $V_{U}$ are orthogonal with respect to $<,>$.

It is easy to verify that $\bar{X}_{1}^{h}, \bar{X}_{2}^{h}, \ldots, \bar{X}_{n}^{h}, \bar{X}_{1}^{v}, \bar{X}_{2}^{v}, \ldots, \bar{X}_{n}^{v}$ are orthonormal vector fields on $T M$ if so are $X_{1}, X_{2}, \ldots, X_{n}$ on $M$, i.e. $g\left(X_{i}, X_{j}\right)=\delta_{j}^{i}$.

$\mathbf{2}^{\mathbf{0}}$. We define a tensor field $J$ on $T M$ by the equalities

$$
J \bar{X}^{h}=\bar{X}^{\nu}, J \bar{X}^{\nu}=-\bar{X}^{h}, X \in \chi(M) .
$$

Since $J^{2}=-I$ and $<J \bar{X}, J \bar{Y}>=<\bar{X}, \bar{Y}>,(T M, J,<,>)$ is an almost Hermitian manifold.

Further, we want to analyze the second fundamental tensor field $h$ of the pari $(J,<,>)$, see (2.11) below, cf. [2]. It is obvious that $(J,<,>)$ is a Kählerian structure if and only if $h=0$.

The Riemannian connection $\hat{\nabla}$ of the metric $\hat{g}=<,>$ on $T M$ is defined by the formula (see [7])

$$
\begin{aligned}
<\hat{\nabla}_{\bar{X}} \bar{Y}, \bar{Z}>= & \frac{1}{2}(\bar{X}<\bar{Y}, \bar{Z}>+\bar{Y}<\bar{Z}, \bar{X}> \\
& -\bar{Z}<\bar{X}, \bar{Y}>+<\bar{Z},[\bar{X}, \bar{Y}]>+<\bar{Y},[\bar{Z}, \bar{X}]> \\
& +<\bar{X},[\bar{Z}, \bar{Y}]>), \quad X, Y, Z \in \chi(M) .
\end{aligned}
$$


For orthonormal vector fields $\bar{X}, \bar{Y}, \bar{Z}$ on $T M$ we obtain

$$
\begin{aligned}
h_{\bar{X} \bar{Y} \bar{Z}}=<h_{\bar{X}} \bar{Y}, \bar{Z}> & =\frac{1}{2}<\hat{\nabla}_{\bar{X}} \bar{Y}+J \hat{\nabla}_{\bar{X}} J \bar{Y}, \bar{Z}> \\
& =\frac{1}{2}\left(<\hat{\nabla}_{\bar{X}} \bar{Y}, \bar{Z}>-<\hat{\nabla}_{\bar{X}} J \bar{Y}, J \bar{Z}>\right) \\
& =\frac{1}{4}(<[\bar{X}, \bar{Y}], \bar{Z}>+<[\bar{Z}, \bar{X}], \bar{Y}>+<[\bar{Z}, \bar{Y}], \bar{X}> \\
& -<[\bar{X}, J \bar{Y}], J \bar{Z}>-<[J \bar{Z}, \bar{X}], J \bar{Y}>-<[J \bar{Z}, J \bar{Y}], \bar{X}>) .
\end{aligned}
$$

Using (2.4)-(2.7) and (2.11) we consider the following cases for the tensor field $h$ assuming all the vector fields to be orthonormal.

$$
\begin{aligned}
& h_{\bar{X}^{h} \bar{Y}^{h} \bar{Z}^{h}}= \frac{1}{4}\left(<\left[\bar{X}^{h}, \bar{Y}^{h}\right], \bar{Z}^{h}>+<\left[\bar{Z}^{h}, \bar{X}^{h}\right], \bar{Y}^{h}>\right. \\
&+<\left[\bar{Z}^{h}, \bar{Y}^{h}\right], \bar{X}^{h}>-<\left[\bar{X}^{h}, J \bar{Y}^{h}\right], J \bar{Z}^{h}>-<\left[J \bar{Z}^{h}, \bar{X}^{h}\right], J \bar{Y}^{h}> \\
&\left.-<\left[J \bar{Z}^{h}, J \bar{Y}^{h}\right], \bar{X}^{h}>\right)=\frac{1}{4}(g([X, Y], Z)+g([Z, X], Y) \\
&+g([Z, Y], X)-<\left[\bar{X}^{h}, \bar{Y}^{\nu}\right], \bar{Z}^{\nu}>-<\left[\bar{Z}^{\nu}, \bar{X}^{h}\right], \bar{Y}^{\nu}> \\
&\left.-<\left[\bar{Z}^{\nu}, \bar{Y}^{\nu}\right], \bar{X}^{h}>\right)=\frac{1}{2} g\left(\nabla_{X} Y, Z\right)-\frac{1}{4}\left(g\left(\nabla_{X} Y, Z\right)\right. \\
&\left.-g\left(\nabla_{X} Z, Y\right)\right)=\frac{1}{2}\left(g\left(\nabla_{X} Y, Z\right)-g\left(\nabla_{X} Y, Z\right)\right)=0 .
\end{aligned}
$$

$\left.2.1^{0}\right) h_{\bar{X}^{h} \bar{Y}^{h} \bar{Z}^{\nu}}=\frac{1}{4}\left(<\left[\bar{X}^{h}, \bar{Y}^{h}\right], \bar{Z}^{\nu}>+<\left[\bar{Z}^{\nu}, \bar{X}^{h}\right], \bar{Y}^{h}>\right.$

$$
\begin{gathered}
+<\left[\bar{Z}^{\nu}, \bar{Y}^{h}\right], \bar{X}^{h}>-<\left[\bar{X}^{h}, J \bar{Y}^{h}\right], J \bar{Z}^{\nu}>-<\left[J \bar{Z}^{\nu}, \bar{X}^{h}\right], J \bar{Y}^{h}> \\
\left.-<\left[J \bar{Z}^{\nu}, J \bar{Y}^{h}\right], \bar{X}^{h}>\right)=\frac{1}{4}(g(R(X, Y) U, Z) \\
\left.+<\left[\bar{Z}^{h}, \bar{X}^{h}\right], \bar{Y}^{\nu}>\right)=\frac{1}{4}(g(R(X, Y) U, Z)+g(R(Z, X) U, Y)) \\
=-\frac{1}{4}(g(R(X, Y) Z, U)+g(R(Z, X) Y, U)) .
\end{gathered}
$$

By similar arguments we obtain

$\left.3.1^{0}\right) h_{\bar{X}^{h} \bar{Y}^{\nu} \bar{Z}^{h}}=-\frac{1}{4}(g(R(Z, X) Y, U)+g(R(X, Y) Z, U))$.

$\left.4.1^{0}\right) h_{\bar{X}^{\nu} \bar{Y}^{h} \bar{Z}^{h}}=-\frac{1}{4}(g(R(Z, Y) X, U))$.

$\left.5.1^{0}\right) h_{\bar{X}^{\nu} \bar{Y}^{\nu} \bar{Z}^{\nu}}=\frac{1}{4}(g(R(Z, Y) X, U))$.

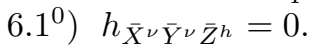

$\left.7.1^{0}\right) h_{\bar{X}^{\nu} \bar{Y}^{h} \bar{Z}^{\nu}}=0$.

$\left.8.1^{0}\right) h_{\bar{X}^{h} \bar{Y}^{\nu} \bar{Z}^{\nu}}=0$.

Thus the second fundamental tensor field $h$ (of the structure $(J,<,>$ )) strongly depends on the connection $\nabla$.

$\mathbf{3}^{\mathbf{0}}$. We have constructed the almost Hermitian structure $(J, \hat{g}=<,>)$ on $T M$ with the help of a Riemannian metric $g$ on $M$, i.e. we have obtained an injective mapping

$$
E:(M) \rightarrow(T M): g \mapsto(J, \hat{g}=<,>),
$$

where $(M)$ is the set of Riemannian metrics on $M$ and $(T M)$ is the set of almost Hermitian structures on $T M$.

Definition 2. A smooth manifold $M$ will be called an $\varepsilon$-manifold if there exists a normal tubular neighborhood $T b(M, \varepsilon)$ in $T M$ with respect to $\hat{g}=<,>=E(g)$ on $T M$ induced by some $g \in(M)$, where $M$ is considered as the null section $O_{M}$ in $T M\left(M \ni p \leftrightarrow O_{p} \in\right.$ $\left.O_{M} \subset T M\right)$. 
It is clear that any smooth compact manifold is an $\varepsilon$-manifold.

THEOREM 3. Let $M$ be an $\varepsilon$-manifold and $\operatorname{Tb}(M, \varepsilon)$ be the corresponding normal tubular neighborhood in TM with respect to $\hat{g}=<,>=E(g)$ on TM. Then $M\left(O_{M}\right)$ is a totally geodesic submanifold of the Kählerian manifold $\left(\operatorname{Tb}\left(M, \frac{\varepsilon}{2}\right), \bar{J}, \bar{g}\right)$, where the almost Hermitian structure $(\bar{J}, \bar{g})$ is the deformation of the structure $(J,<,>)$ defined above.

Proof. It follows from Theorem 1 that $M$ is a totally geodesic submanifold of the Riemannian manifold $\left(T b\left(M, \frac{\varepsilon}{2}\right), \bar{g}\right)$.

Let $\tilde{W}_{0}$ be a coordinate neighborhood in $T M$ considered in $\mathbf{1}^{\mathbf{0}}, \mathbf{1}$. A point $x \in \tilde{W}_{0}$ has the coordinates $x_{1}, \ldots, x_{n}, x_{n+1}, \ldots, x_{2 n}$ where $x_{1}, \ldots, x_{n}$ are coordinates of the point $p$ in $\tilde{V}_{0} \subset M$ and $x_{n+1}, \ldots, x_{2 n}$ are normal coordinates of $x$ in $D\left(p ; \frac{\varepsilon}{2}\right)$.

Let denote $X_{i}=\frac{\partial}{\partial x_{i}}, i=\overline{1,2 n}, \hat{\nabla}_{X_{i}} X_{j}=\sum_{k} \hat{\Gamma}_{i j}^{k} X_{k}, \bar{\nabla}_{X_{i}} X_{j}=\sum_{k} \bar{\Gamma}_{i j}^{k} X_{k}, J X_{j}=$ $\sum_{k} J_{j}^{k} X_{k}, \bar{J} X_{j}=\sum_{k} J_{j}^{k} X_{k}, \hat{g}_{i j}=\hat{g}\left(X_{i}, X_{j}\right), \bar{g}_{i j}=\bar{g}\left(X_{i}, X_{j}\right)$ where $\hat{\nabla}$ and $\bar{\nabla}$ are Riemannian connections of metrics $\hat{g}$ and $\bar{g}$, respectively.

Using the construction of $\mathbf{2}^{\mathbf{0}}, \mathbf{1}$ we have $\bar{g}_{i j}(x)=\hat{g}_{i j}(p), \bar{J}_{j}^{i}(x)=J_{j}^{i}(p)$ on $T b\left(M, \frac{\varepsilon}{2}\right) \cap$ $\tilde{W}_{0}$. According to [7] we can write

$$
\sum_{l} \bar{g}_{l k} \bar{\Gamma}_{i j}^{l}=\frac{1}{2}\left(\frac{\partial \bar{g}_{k j}}{\partial x_{i}}+\frac{\partial \bar{g}_{i k}}{\partial x_{j}}-\frac{\partial \bar{g}_{i j}}{\partial x_{k}}\right) .
$$

It follows from $(2.12)$ that $\bar{\Gamma}_{i j}^{l}(x)=\bar{\Gamma}_{i j}^{l}(p)$ and $\bar{\Gamma}_{i j}^{l}(x)=0$, i.e. $\bar{\nabla}_{X_{i}} X_{j}=0$ for $i=$ $\overline{n+1,2 n}$.

Further, we get

$$
\begin{gathered}
\left(\bar{\nabla}_{X_{i}} \bar{J}\right) X_{j}=\bar{\nabla}_{X_{i}} \bar{J} X_{j}-\bar{J} \bar{\nabla}_{X_{i}} X_{j}=\sum_{k} \bar{\nabla}_{X_{i}} \bar{J}_{j}^{k} X_{k} \\
-\bar{J}\left(\sum_{k} \bar{\Gamma}_{i j}^{k} X_{k}\right)=\sum_{k}\left(\bar{J}_{j}^{k} \bar{\nabla}_{X_{i}} X_{k}+\left(X_{i} \bar{J}_{j}^{k}\right) X_{k}\right) \\
-\sum_{k, l} \bar{\Gamma}_{i j}^{l} \bar{J}_{l}^{k} X_{k}=\sum_{k, l}\left(\bar{J}_{j}^{l} \bar{\Gamma}_{i l}^{k}-\bar{\Gamma}_{i j}^{l} \bar{J}_{l}^{k}+X_{i} \bar{J}_{j}^{k}\right) X_{k}, \\
\left(\left(\bar{\nabla}_{X_{i}} \bar{J}\right) X_{j}\right)(x)=\sum_{k, l}\left(\bar{J}_{j}^{l} \bar{\Gamma}_{i l}^{k}-\bar{\Gamma}_{i j}^{l} \bar{J}_{l}^{k}+X_{i} \bar{J}_{j}^{k}\right)(x) X_{k \mid x} \\
=\sum_{k, l}\left(\left(\bar{J}_{j}^{l} \bar{\Gamma}_{i l}^{k}-\bar{\Gamma}_{i j}^{l} \bar{J}_{l}^{k}\right)(p)+\left(X_{i} \bar{J}_{j}^{k}\right)(x)\right) X_{k \mid x} .
\end{gathered}
$$

It follows that $\bar{\nabla}_{X_{i}} \bar{J}=0$ for $i=\overline{n+1,2 n}$. For $i=\overline{1, n},\left(X_{i} \bar{J}_{j}^{k}\right)(x)=\left(X_{i} J_{j}^{k}\right)(p)$ and we obtain

$$
\left(\left(\bar{\nabla}_{X_{i}} \bar{J}\right) X_{j}\right)(x)=\sum_{k, l}\left(J_{j}^{l} \hat{\Gamma}_{i l}^{k}-\hat{\Gamma}_{i j}^{l} J_{l}^{k}+X_{i} J_{j}^{k}\right)(p) X_{k \mid x} .
$$

On the other hand we can write

$$
\left(\left(\hat{\nabla}_{X_{i}} J\right) X_{j}\right)(p)=\sum_{k, l}\left(J_{j}^{l} \hat{\Gamma}_{i l}^{k}-\hat{\Gamma}_{i j}^{l} J_{l}^{k}+X_{i} J_{j}^{k}\right)(p) X_{k \mid p} .
$$

According to [2], we have $\left(\left(\nabla_{X_{i}} J\right) X_{j}\right)(p)=\left(2 h_{X_{i}} J X_{j}\right)(p)$ where the tensor field $h$ is defined by (2.11). From $\left.\left.1 \cdot 1^{0}\right)-8 \cdot 1^{0}\right)$ it follows that $h_{p}=0$ for any $p \in M^{\prime}\left(U=O_{p} \in O_{M}\right)$. 
Finally, we have obtained that $\bar{\nabla} \bar{J}=0$ and the structure $(\bar{J}, \bar{g})$ is a Kählerian one on $\operatorname{Tb}\left(M, \frac{\varepsilon}{2}\right)$.

COROllary 3. Any compact smooth manifold of dimension $n$ can be embedded in a Kählerian manifold of dimension $2 n$ as a totally geodesic submanifold.

$4^{0}$. Let $M$ be an arbitrary smooth Riemannian manifold. For any point $p \in M$ we can consider a set $\{\delta(p)\}$ of positive numbers such that the mapping $\exp _{\mid U(\delta(p))}$ is defined and injective on $U(\delta(p)) \subset T_{p} M$. Let $\varepsilon(p)=\sup \{\delta(p)\}$.

Lemma 4 ([6]). The mapping $M \rightarrow \boldsymbol{R}_{+}: p \mapsto \varepsilon(p)$ is continuous on $M$.

From the constructions above it is evident that the following proposition is true.

Proposition 5. There exists a function $\varepsilon(p)$ continuous on $M$ such that the subset $T b(M, \varepsilon(p))=\bigcup_{p \in M} D(p ; \varepsilon(p))$ of $T M$ defined by the Riemannian metric $\widehat{g}=E(g)$ on $T M$ induced by some $g \in R(M)$ is a normal tubular neighborhood of $M$ considered as the null section $O_{M}$ of $T M$.

All the constructions considered above for the case $\varepsilon=$ const can be generalized to the case of a positive continuous function $\varepsilon(p)$ on $M$.

THEOREM 6. Let $M$ be a smooth real manifold and $T b(M, \varepsilon(p))$ be the corresponding normal tubular neighborhood in TM with respect to some $\widehat{g}=E(g)$ on $T M$. Then $M\left(O_{M}\right)$ is a totally geodesic submanifold of the Kählerian manifold $\left(T b\left(M, \frac{\varepsilon(p)}{2}, \bar{J}, \bar{g}\right)\right)$, where the almost Hermitian structure $(\bar{J}, \bar{g})$ is the deformation of the structure $(J, \widehat{g})$. So, any smooth real manifold of dimension $n$ can be embedded into a Kählerian manifold of dimension $2 n$ as a totally geodesic submanifold.

\section{Deformations of structures on a neighborhood of a curve and geometric antigravitation}

$\mathbf{1}^{\mathbf{0}}$. Let $(M, g)$ be a Riemannian smooth $n$-dimensional manifold and $\gamma: p=p(s), 0 \leq$ $s \leq s_{1}$, be a smooth curve isometrically embedded in $M$. We can consider a balloon neighborhood $\operatorname{Bl}(\gamma, \varepsilon)$ consisting of the tubular neighborhood $\operatorname{Tb}(\gamma, \varepsilon)$ and two geodesic semiballs $B s(p(0) ; \varepsilon), B s\left(p\left(s_{1}\right) ; \varepsilon\right)$ attached to $T b(\gamma, \varepsilon)$ at the ends. For any point $x \in$ $T b(\gamma, \varepsilon)$, there exist $0 \leq s \leq s_{1}$ and $0 \leq t<\varepsilon(p(s))$ such that $x=\exp _{p(s)}(t \xi)$, where $\|\xi\|=1, g(\xi, \nu)=0$, and $\nu=\dot{p}(s)$. For every point $x \in B s(p(0) ; \varepsilon)\left(\right.$ or $x \in B s\left(p\left(s_{1}\right) ; \varepsilon\right)$ ) we have $x=\exp _{p_{0}}(t \xi),\|\xi\|=1, \xi \in T_{p_{0}} M, p_{0}=p(0)$ (or $x=\exp _{p_{1}}(t \xi),\|\xi\|=1$, $\left.\xi \in T_{p_{1}} M, p_{1}=p\left(s_{1}\right)\right)$. We can choose local coordinates $\left(x_{1}, x_{2}, \ldots, x_{n}\right)$ on $\operatorname{Bl}(\gamma, \varepsilon)$ such that $\left.\frac{\partial}{\partial x_{1}}\right|_{p}=\nu_{p}$. We denote $X_{i_{x}}=\left.\frac{\partial}{\partial x_{i}}\right|_{x}, i=1,2, \ldots, n$.

$\mathbf{2}^{\mathbf{0}}$. Let $K$ be a tensor field of type $(r, s)$ from the algebra of all smooth tensor fields on $M$ and on $\operatorname{Bl}(\gamma, \varepsilon)$ we have

$$
K_{x}=\sum_{i_{1}, \ldots, i_{r}, j_{1}, \ldots, j_{s}} k_{j_{1}, \ldots, j_{s}}^{i_{1}, \ldots, i_{r}}(x) X_{i_{1}, x} \otimes \cdots \otimes X_{i_{r}, x} \otimes X_{x}^{j_{1}} \otimes \cdots \otimes X_{x}^{j_{s}}
$$

where $\left\{X_{x}^{1}, \ldots, X_{x}^{n}\right\}$ is the dual basis of $T_{x}^{*} M$.

A tensor field $\bar{K}$ is defined on $M$ as follows: 
a) $x \in B l\left(\gamma ; \frac{\varepsilon}{4}\right)$,

$$
\bar{K}_{x}=\sum_{i_{1}, \ldots, i_{r}, j_{1}, \ldots, j_{s}} k_{j_{1}, \ldots, j_{s}}^{i_{1}, \ldots, i_{r}}\left(p_{0}\right) X_{i_{1}} \otimes \cdots \otimes X_{i_{r_{x}}} \otimes X_{x}^{j_{1}} \otimes \cdots \otimes X_{x}^{j_{s}} ;
$$

b) $x \in B l\left(\gamma ; \frac{\varepsilon}{2}\right) \backslash B l\left(\gamma ; \frac{\varepsilon}{4}\right), x=\exp _{p}(t \xi), p=p\left(s_{p}\right)$,

$$
\begin{array}{r}
\bar{K}_{x}=\sum_{i_{1}, \ldots, i_{r}, j_{1}, \ldots, j_{s}} k_{j_{1}, \ldots, j_{s}}^{i_{1}, \ldots, i_{r}}\left(p\left(\frac{2 s_{p}}{\varepsilon}\left(2 t-\frac{\varepsilon}{2}\right)\right)\right) X_{i_{1_{x}}} \\
\otimes \cdots \otimes X_{i_{r_{x}}} \otimes X_{x}^{j_{1}} \otimes \cdots \otimes X_{x}^{j_{s}}
\end{array}
$$

c) $x \in B l(\gamma, \varepsilon) \backslash B l\left(\gamma, \frac{\varepsilon}{2}\right), x=\exp _{p}(t \xi), p=p\left(s_{p}\right)$,

$$
\begin{array}{r}
\bar{K}_{x}=\sum_{i_{1}, \ldots, i_{r}, j_{1}, \ldots, j_{s}} k_{j_{1}, \ldots, j_{s}}^{i_{1}, \ldots, i_{r}}\left(\exp _{p}((2 t-\varepsilon) \xi)\right) X_{i_{1_{x}}} \\
\otimes \cdots \otimes X_{i_{r_{x}}} \otimes X_{x}^{j_{1}} \otimes \cdots \otimes X_{x}^{j_{s}} ;
\end{array}
$$

d) $x \in M \backslash B l(\gamma, \varepsilon)$,

$$
\bar{K}_{x}=K_{x}
$$

REMARK. The obtained tensor field $\bar{K}$ is continuous but is not smooth on the boundaries of the balloon neighborhoods, $\bar{K}$ is smooth at other points of M.

Problem. Can we get a good deformation $\tilde{K}$ of $\bar{K}$ such that $\tilde{K}$ is smooth and $\tilde{K}=\bar{K}$ on some balloon neighborhood of $\gamma, \tilde{K}=\bar{K}$ on $M \backslash B l(\gamma, \varepsilon)$ ?

$\mathbf{3}^{\mathbf{0}}$. Let $\Phi(X, Y)=g(P X, Y)$ be a pseudo-Riemannian metric on $M, P^{2}=I, X, Y \in$ $\chi(M)$. Using the construction presented in $\mathbf{2}^{\mathbf{0}}$ we can obtain the deformations $\bar{g}$ of $g$ and $\bar{P}$ of $P$ on $B l(\gamma, \varepsilon)$, i.e. a new pseudo-Riemannian metric $\bar{\Phi}$ where $\bar{\Phi}(X, Y)=$ $\bar{g}(\bar{P} X, Y), \bar{P}^{2}=I$.

THEOREM 7. Let $\bar{\nabla}$ be the canonical connection of $\bar{\Phi}$ on $\operatorname{Bl}\left(\gamma ; \frac{\varepsilon}{4}\right)$. Then $\gamma$ is geodesic with respect to $\bar{\nabla}$ and the curvature tensor field (gravitation) $\bar{R}$ vanishes on $\operatorname{Bl}\left(\gamma ; \frac{\varepsilon}{4}\right)$.

Proof. For any $x \in B l\left(\gamma ; \frac{\varepsilon}{4}\right)$ we have

$$
\bar{g}_{x}=\sum_{j, k} g_{j k}\left(p_{0}\right) X_{x}^{j} \otimes X_{x}^{k}, \quad \bar{P}_{x}=\sum_{i, l} f_{l}^{i}\left(p_{0}\right) X_{i_{x}} \otimes X_{x}^{l}
$$

and the functions $g_{j k}\left(p_{0}\right), f_{l}^{i}\left(p_{0}\right)$ are constant on $B l\left(\gamma ; \frac{\varepsilon}{4}\right)$. The formula of the canonical connection $\bar{\nabla}$ of $\bar{\Phi}$ is similar to $(1.1)$

$$
\begin{aligned}
2 \bar{\Phi}_{x} & \left(\bar{\nabla}_{X_{i}} X_{j}, X_{l}\right)=X_{i_{x}} \bar{\Phi}\left(X_{j}, X_{l}\right)+X_{j_{x}} \bar{\Phi}\left(X_{i}, X_{l}\right)-X_{l_{x}} \bar{\Phi}\left(X_{i}, X_{j}\right) \\
& +\bar{\Phi} x\left(\left[X_{i}, X_{j}\right], X_{l}\right)+\bar{\Phi} x\left(\left[X_{l}, X_{i}\right], X_{j}\right)+\bar{\Phi} x\left(X_{i},\left[X_{l}, X_{j}\right]\right) \\
& =X_{i_{x}} \bar{g}\left(\bar{P} X_{j}, X_{l}\right)+X_{j_{x}} \bar{g}\left(\bar{P} X_{i}, X_{l}\right)-X_{l_{x}} \bar{g}\left(\bar{P} X_{i}, X_{j}\right)=0 .
\end{aligned}
$$

So we obtain that $\bar{\nabla}_{X_{i}} X_{j}=0$ and it follows that $\bar{R}=0$ on $B l\left(\gamma ; \frac{\varepsilon}{4}\right)$. From $\bar{\nabla}_{X_{1}} X_{1}=0$ we get that $\gamma$ is a geodesic segment.

Remark. We can also consider almost Hermitian manifolds and Riemannian G-structures, see [3] for details. 


\section{References}

[1] P. Dombrowski, On the geometry of the tangent bundle, J. Reine Angew. Math. 210 (1962), 73-88.

[2] A. A. Ermolitski, Riemannian manifolds with geometric structures, BSPU, Minsk, 1998 (in Russian).

[3] A. A. Ermolitski, Theorem about hat and problems of classification of structures on Riemannian manifolds, Izv. Vuzov Mat. 11 (2002) 27-31 (in Russian).

[4] A. A. Ermolitski, Deformations of distributions on Riemannian manifolds, in: 5th International Conference "Geometry and Topology of Manifolds" (Krynica), Abstracts, Łódź, 2003, 26-28.

[5] A. A. Ermolitski, Deformations of structures on a neighbourhood of a curve and geometric antigravitation, in: 6th International Conference "Geometry and Topology of Manifolds" (Krynica), Abstracts, Łódź, 2004, 59-60.

[6] D. Gromoll, W. Klingenberg and W. Meyer, Riemannsche Geometrie im Grossen, Springer, Berlin, 1968.

[7] S. Kobayashi and K. Nomizu, Foundations of Differential Geometry, Vol. 1, Wiley, 1963.

[8] S. Kobayashi and K. Nomizu, Foundations of Differential Geometry, Vol. 2, Wiley, 1969. 